\title{
Selecting the Optimal Morse Falls Scale Cut-Off Point for Patient Fall Risk
}

\author{
Sikha Bagui ${ }^{1}$ (D), Tyler Long1, Subhash Bagui² \\ ${ }^{1}$ Department of Computer Science, University of West Florida, Pensacola, FL, USA \\ ${ }^{2}$ Department of Mathematics and Statistics, University of West Florida, Pensacola, FL, USA \\ Email: bagui@uwf.edu,t150@students.uwf.edu,sbagui@uwf.edu
}

How to cite this paper: Bagui, S., Long, T. and Bagui, S. (2019) Selecting the Optimal Morse Falls Scale Cut-Off Point for Patient Fall Risk. Health, 11, 924-931.

https://doi.org/10.4236/health.2019.117074

Received: June 21, 2019

Accepted: July 21, 2019

Published: July 24, 2019

Copyright $\odot 2019$ by author(s) and Scientific Research Publishing Inc. This work is licensed under the Creative Commons Attribution International License (CC BY 4.0).

http://creativecommons.org/licenses/by/4.0/

\begin{abstract}
Patient falls have long been a difficult problem and topic of research in the inpatient hospital environment. Hospitals experience heavy financial burden due to patient falls. Preventing falls can improve patient outcomes as well as reduce financial burden on both the patient as well as the healthcare organization. In this study, we determine the optimal cut-off value for patients at risk for falls using a Falls Screening Tool, the Morse Falls Scale. This study was conducted at a community-owned hospital with 256 acute care private rooms and 36 critical care rooms. This study used only the admission Morse Falls Scale (MFS) score in its analysis.
\end{abstract}

\section{Keywords}

Patient Falls, Morse Falls Scale, Preventing Falls, Falls Risk Assessment, Falls Prevention

\section{Introduction}

Patient falls have long been a difficult problem and a topic of research in the inpatient hospital environment. Hospitals experience a financial burden due to patient falls. Studies have found that inpatient falls occur at a rate of $3-5$ per 1000 inpatient days and that roughly one-third of patient falls result in injury, which can range from minor injury to death [1]. Falls are associated with an increase in both the length of time a patient must stay in the hospital as well as the patient's cost of care [2]. Patients that experience a fall in the hospital have an increase in length of stay, on the average, of eight days, and an average increased cost of $\$ 6669$. Patients for whom the fall results in an injury have an average additional four days in the hospital (for a total of 12 days compared to non-fallers) and an average additional cost of $\$ 4727$ (for a total of $\$ 11,396$ compared to non-fallers) 
[2].

Since inpatient falls are designated as "adverse hospital events" [3], a categorization given to negative events deemed as preventable, any additional costs associated with a patient's care are not covered by most insurance companies-meaning that hospitals must provide more care without any additional reimbursement. There are also costs originating from lawsuits filed by the patients and families of patients that suffer a fall while in the hospital. Considering that inpatient falls are the most frequent incident reported by hospitals [1], they are a prevalent and expensive problem. A reduction in patient falls can provide benefits both financially and otherwise, that is, with timely access to health care services.

A large amount of research has been conducted in an attempt to better understand the causes of falls and the possible interventions that may prevent their occurrence or reduce the resulting harm. Many accreditation and quality improvement organizations such as The Joint Commission and The Agency for Healthcare Research and Quality (AHRQ) highlight the issue and have ongoing efforts to reduce harm from falls [4] [5]. Preventing falls can improve patient outcomes and reduce financial burden to healthcare organizations.

One of the first steps in a fall prevention program is to select and implement a fall-risk screening tool. Many such tools exist, more than 35 according to one study [6]. Three of the most common screening tools, as they have been validated in multiple studies with various populations, are the St Thomas's Risk Assessment Tool in Falling Elderly Inpatients (STRATIFY), Morse Fall Scale (MFS), and the Hendrich Fall Risk Model (HFRM) [6].

In this study, we use the Morse Fall Score to determine the optimal cut-off value at which patients are at risk for falls.

\subsection{What Is a Patient Fall?}

Defining what constitutes a patient fall can be a somewhat complex question to answer. Multiple definitions for what constitutes a patient fall exist. They take into account the various internal and external factors that may cause a fall to occur, as well as the level of contact required with surfaces, objects, or people to be counted as a fall [1]. The facility at which this analysis was conducted uses the definition provided by The National Database of Nursing Quality Indicators (NDNQI). It states, "A patient fall is a sudden, unintentional descent, with or without injury to the patient, that results in the patient coming to rest on the floor, on or against some other surface (e.g., a counter), on another person, or on an object (e.g., a trash can)" [7].

\subsection{Fall Risk Assessment}

The best screening tool to use depends on the unique characteristics of each health care organization. The Morse Falls Scale (MFS) is one of the most prevalent tools used to assess a patients' fall risk. The MFS provides a total risk score 
ranging between 0 and 125-where a cut-off value is used to determine if a patient is at risk for a fall and therefore fall prevention interventions should be initiated. The optimal cut-off value ranges between of 25 - 55, with the standard cut-off value of 45 suggested by the creators of the tool [8]. Studies have found different cut-off values to be optimal in different organizations [9] [10]. The organization in which this analysis was conducted found the cut-off value of 45 to be optimal based on a statistical analysis of the specificity and sensitivity of each potential cut off value.

\subsection{The Morse Falls Scale}

The Morse Falls Scale (MFS) is one of the most prevalent tools used to assess a patients' fall risk. The MFS is composed of six sub-items: 1) history of falling, 2) secondary diagnosis, 3) ambulatory aids, 4) intravenous therapy, 5) type of gait, and 6) mental status. The responses to each of the sub-items are assigned values, which are summed up to get a total risk score ranging between 0 and 125. Finally, a cut-off value is used to determine if a patient is at risk for a fall and therefore should receive fall prevention interventions. The standard cut-off value of $>45$ is proposed, yet the developer of the scale suggests that an optimal cut-off value will vary by organization. As such, an analysis should be conducted to determine the optimal cut-off value within the range of 25 - 55 [8]. The facility at which this analysis was conducted had used the MFS for approximately 2 years at the time of this study. However, the optimal cut-off value for fall risk had not been determined.

\section{Related Works}

Many studies have been conducted seeking to study the effectiveness of using the MFS and other fall-risk assessment tools on identifying the patients that are more likely to fall. [6] [11] discuss research in which the effectiveness was tested of the three most common tools (MFS, STRATIFY, and HFRM). The results showed that different tools proved better in different organizations. These tools also have varying qualities that should be evaluated to determine appropriateness of a tool to a specific organization. The conclusion was that each of these tools should be evaluated for effectiveness at the outset of a fall prevention program prior to selecting a fall-risk assessment tool.

Additionally, studies have been conducted to find the optimal cut-off value of the MFS to categorize a patient as at risk for falling. [9] looked at the predictive value of the MFS at various cut-off points and found 55 to be the optimal cut-off point at their organization. [10], on the other hand, conducted a similar analysis and found 51 to be the optimal cut-off point.

Finally, [12] demonstrated an improvement upon the predictive value of a fall-risk assessment by including additional clinical data collected from the hospital's electronic medical record system. Certain data points that can easily be queried in the age of electronic medical records-such as diagnoses, test results, 
and administered medications-allowed this organization to achieve a better risk-model than a fall-risk assessment tool alone.

\section{Method}

\subsection{Data Source and Population Description}

This study was conducted at a community-owned hospital with 256 acute care private rooms and 36 critical care rooms. Patients were assessed for their MFS by a nurse upon admission and then every shift thereafter until discharge. This study used only the admission MFS score in its analysis. Before beginning the analysis of cut-off values, we verified that a significant difference existed in the admission MFS score between patients that experienced one or more falls and those that did not (Figure 1). In Figure 1, the pooled standard deviation is used to calculate the intervals.

Our study did not require approval by the Internal Review Board as it was a quality improvement activity with no patient interaction and was conducted on de-identified patient data.

The data set for our study included 1000 patients out of a population of 13,626 that met the following criteria. Patients were 18 years of age or older, admitted to and discharged from an inpatient unit between 01/01/2018 - 02/28/2019, and did not spend any time in behavioral health areas. Inpatient units included the following specialties: Medical, Surgical, Orthopedics, Oncology, Neurosciences, Cardiac, Mother-Baby, Surgical Intensive Nursing Care, Cardiovascular Intensive Critical Care, and Progressive Care. Of those meeting the criteria, there were 207 patients identified by the hospital's adverse event reporting system who experienced one or more falls. The additional 793 patients, who were not reported

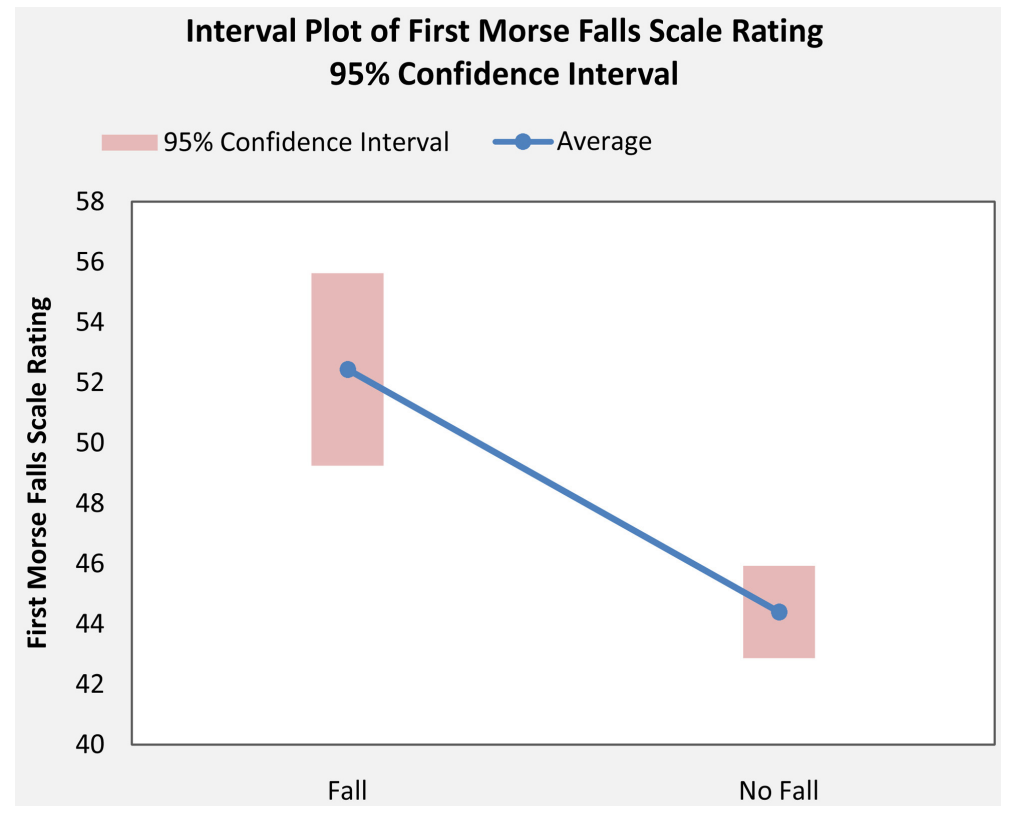

Figure 1. Interval plot of first morse falls scale rating at 95\% confidence interval. 
to have fallen, were randomly selected from the population. The demographics for the falls patients is: patient age (mean: 64.13, SD: 15.75); patient gender (Male 109, 52.66\%; Female 98, 47.34\%); and the first recorded MFS Rating of the patient's visit (mean: 52.44, SD: 23.40). The demographics for the patients who were not reported to have fallen is: patient age (mean: 62.39, SD: 17.45); patient gender (Male 394, 49.68\%; Female 399, 50.32\%); and the first recorded MFS Rating of the patient's visit (mean: 44.39, SD: 21.99). And, the demographics for the total sample is patient age (mean: 62.75, SD: 17.12); patient gender (Male 503, 50.3\%; Female 497, 49.7\%); and the first recorded MFS Rating of the patient's visit (mean: 46.06, SD: 22.52).

Cut-off points from 20 - 70 in five-point intervals were evaluated. The following statistics were calculated for each cut-off point: sensitivity, specificity, positive predictive value (PPV), negative predictive value (NPV), Accuracy, and area under the curve (AUC) (Table 1). A receiver-operating characteristic curve (ROC curve) was drawn to evaluate the optimal cut-off value (Figure 2). A description of each of these statistics as it relates to the sample follows.

\subsection{Statistical Analysis}

Sensitivity represents the proportion of the total number of patients that fell in the sample that were determined as at risk for falling at each cut-off point-also known as the true positive rate. Specificity represents the proportion of the total number of patients that did not fall that were determined as not at risk for falling at each cut-off point-also known as the true negative rate. The PPV shows the proportion of the total number of patients determined as at risk for falling that experienced a fall at each cut-off point. The NPV shows the proportion of the total number of patients determined as not at risk for falling that did not experience a fall at each cut-off point. Accuracy represents the proportion of the number of patients at risk for falling that fell and the number of patients not at risk for falling that did not fall to the total number of patients in the sample. The AUC at each cut-off value can be calculated as either an unweighted or a

Table 1. Statistical metrics.

\begin{tabular}{ccccccccccc}
\hline Cut-off Value & 20 & 25 & 35 & 40 & 45 & 50 & 55 & 60 & 65 & 70 \\
\hline Sensitivity & $84.1 \%$ & $83.6 \%$ & $64.7 \%$ & $63.8 \%$ & $52.2 \%$ & $46.9 \%$ & $44.4 \%$ & $32.4 \%$ & $31.4 \%$ & $21.7 \%$ \\
Specificity & $24.0 \%$ & $24.6 \%$ & $49.7 \%$ & $50.4 \%$ & $63.4 \%$ & $66.6 \%$ & $69.2 \%$ & $81.6 \%$ & $82.1 \%$ & $87.5 \%$ \\
PPV & $22.4 \%$ & $22.4 \%$ & $25.1 \%$ & $25.1 \%$ & $27.1 \%$ & $26.8 \%$ & $27.4 \%$ & $31.5 \%$ & $31.4 \%$ & $31.3 \%$ \\
NPV & $85.2 \%$ & $85.2 \%$ & $84.4 \%$ & $84.2 \%$ & $83.6 \%$ & $82.8 \%$ & $82.7 \%$ & $82.2 \%$ & $82.1 \%$ & $81.1 \%$ \\
Accuracy & $36.4 \%$ & $36.8 \%$ & $52.8 \%$ & $53.2 \%$ & $61.1 \%$ & $62.5 \%$ & $64.1 \%$ & $71.4 \%$ & $71.6 \%$ & $73.9 \%$ \\
\% High Risk & $77.7 \%$ & $77.1 \%$ & $53.3 \%$ & $52.5 \%$ & $39.8 \%$ & $36.2 \%$ & $33.6 \%$ & $21.3 \%$ & $20.7 \%$ & $14.4 \%$ \\
AUC & & & & & & & & & & \\
(equal weight) & 0.540 & 0.541 & 0.572 & 0.571 & 0.578 & 0.567 & 0.568 & 0.570 & 0.567 & 0.546 \\
AUC (weighted) & 0.364 & 0.368 & 0.528 & 0.532 & 0.611 & 0.625 & 0.641 & 0.714 & 0.716 & 0.739 \\
\hline
\end{tabular}




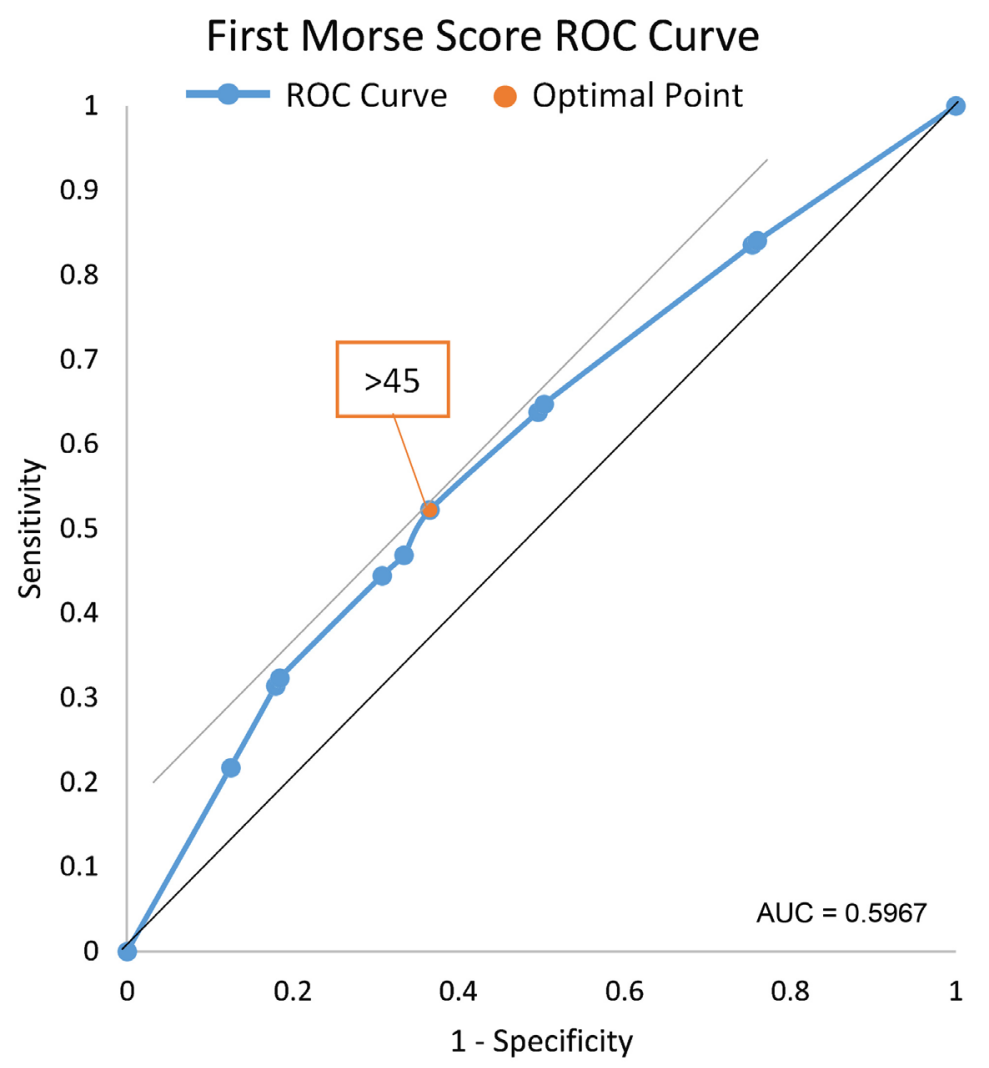

Figure 2. First morse falls score ROC curve.

weighted average of the sensitivity and the specificity. For both sensitivity and specificity, a higher value is better and finding the highest AUC value points to the most favorable tradeoff between these two measures and therefore the optimal MFS cut-off value for an organization. The ROC curve plots the unweighted interaction between sensitivity and specificity to provide a visual representation of the relationship.

The primary metrics that we will use to make our decision about the optimal cut-off value for fall risk is sensitivity, specificity, equal weight AUC, and weighted AUC. There is some subjectivity involved in this sort of analysis such as the predisposition in this case of overestimating rather than underestimating a patients' risk for falling. Over applying fall-prevention strategies to patients that may not need them is better than under applying them, resulting in patient falls that could have been prevented. As such, our analysis looks to maximize the equal-weighted and the weighted AUC while keeping both sensitivity and specificity higher than $50 \%$.

\section{Results}

The proportion of patients that were classified as at risk for falling based on their admission MFS was from $77.8 \%$ (at cut-off $>20$ ) to $14.4 \%$ (at cut-off $>70$ ). The sensitivity ranged from $84.06 \%$ to $21.74 \%$., specificity from $87.52 \%$ to $23.96 \%$, PPV from $31.25 \%$ to $22.39 \%$, NPV from $85.20 \%$ to $81.07 \%$ and accuracy from 
$73.90 \%$ to $36.40 \%$ (Table 1 ). The Area Under the Curve (AUC) using the MFS was 0.5967 (Figure 2).

The optimal cut-off point was identified as $>45$ as it was the point furthest from of the ROC curve representing the best tradeoff between sensitivity and specificity (Figure 2). This value matches the standard cut-off point suggested by Morse [9] and is the cut-off value that is currently in use at this facility. Nevertheless, the ranges of cut-off points from $>35$ to $>65$ were only slightly closer to the diagonal line, the area representing no predictive power. The cut-off value of $>45$ had quite low sensitivity of $52.17 \%$, acceptable specificity of 63.43 , good NPV $83.55 \%$, and $39.8 \%$ of patients in our sample categorized as at risk for falling.

\section{Discussion}

The AUC value of 0.5967 using the MFS, while better than random, suggests a somewhat poor measure of prediction. Our recommendation would be that the facility continue to use the current cut-off value of $>45$ as it has the highest cut-off value AUC (0.578) suggesting the highest predictive power. It also benefits from being the standard cut-off value used with the MFS and therefore may be the most common value used by health care workers as they transition between organizations. If, however, this organization wants to include more patients in a falls prevention protocol, it would be acceptable to move the cut-off value to 40 (Sensitivity $63.77 \%$, Specificity $50.44 \%$ ) or 35 (Sensitivity $64.73 \%$, Specificity $49.68 \%$ ). The tradeoff of this decision, which allows more fall-prone patients to receive clinical intervention, is a higher proportion of patients predicted to fall that would not have fallen without intervention. It would not be recommended to use the cut-off value of 25 (Sensitivity $83.57 \%$, Specificity $24.59 \%$ ) as the specificity is too low to support this decision.

\section{Limitations}

Ideally, a study with this purpose should be conducted prior to implementing a falls prevention program. Since this facility is already using the MFS, the patient care team should be responding to the results of the MFS assessments with appropriate interventions, which in turn would affect the predictive results of the assessment. Good patient care could result in a patient predicted to fall not falling, thus flattening the ROC curve. Additionally, not accurately assessing a patients' MFS value due to either variations between caregivers in the interpretation of the sub-questions of the MFS or haphazardness in its usage due to low perceived value use could result in poor predictive power.

\section{Future Work}

Future work should be conducted to improve the predictive power of fall risk assessments by supplementing the information gained by using a fall-risk assessment tool with data that is captured in the electronic medical record. 


\section{Acknowledgements}

This work has been partially supported by the Askew Institute of the University of West Florida.

\section{Conflicts of Interest}

The authors declare no conflicts of interest regarding the publication of this paper.

\section{References}

[1] Currie, L. (2019) Fall and Injury Prevention. In: Patient Safety and Quality: An Evidence-Based Handbook for Nurses, Agency for Healthcare Research and Quality, Rockville, MD. https://www.ncbi.nlm.nih.gov/books/NBK2653/

[2] Morello, R., et al. (2015) The Extra Resource Burden of in-Hospital Falls: A Cost of Falls Study. The Medical Journal of Australia, 203, 367.

[3] Levinson, D. (2010) Adverse Events in Hospitals: National Incidence among Medicare Beneficiaries. Office of Evaluation and Inspections, Washington DC. https://oig.hhs.gov/oei/reports/oei-06-09-00090.pdf

[4] Alert, S.E. (2015) Preventing Falls and Fall-Related Injuries in Health Care Facilities. The Joint Commission, Sentinel Event Alert, No. 55.

[5] Agency for Healthcare Research \& Quality (2013) Preventing Falls in Hospitals. https://www.ahrq.gov/professionals/systems/hospital/fallpxtoolkit/index.html

[6] Cumbler, E., Simpson, J., Rosenthal, L. and Likosky, D. (2013) Inpatient Falls. The Neurohospitalist, 3, 135-143. https://doi.org/10.1177/1941874412470665

[7] National Database for Nursing Quality Indicators (2016) Guidelines for Data Collection and Submission on Patient Falls Indicator.

[8] Morse, J. (2008) Preventing Patient Falls: Establishing a Fall Intervention Program. Springer Publishing Company, New York.

[9] Schwendimann, R., De Geest, S. and Milisen, K. (2006) Evaluation of the Morse Fall Scale in hospitalised patients. Age and Ageing, 35, 311-313.

https://doi.org/10.1093/ageing/afj066

[10] Baek, S., Piao, J., Jin, Y. and Lee, S. (2013) Validity of the Morse Fall Scale Implemented in an Electronic Medical Record System. Journal of Clinical Nursing, 23, 2434-2441. https://doi.org/10.1111/jocn.12359

[11] Watson, B., Salmoni, A. and Zecevic, A. (2016) The Use of the Morse Fall Scale in an Acute Care Hospital. Clinical Nursing Studies, 4, 32-40. https://doi.org/10.5430/cns.v4n2p32

[12] Gringauz, I., Shemesh, Y., Dagan, A., Israelov, I., et al. (2017) Risk of Falling among Hospitalized Patients with High Modified Morse Scores Could Be Further Stratified. BMC Health Services Research, 17, 721. https://doi.org/10.1186/s12913-017-2685-2 